\title{
The Nature of Light: What Hidden Behind Young's Double-Slit Experiment?
}

\author{
Xiuqing Huang* \\ Department of Telecommunications Engineering ICE, Army Engineering University, Nanjing 210007, China
}

(Dated: February 2, 2021)

\begin{abstract}
We experimentally prove that the famous single- and double-slit experiments are the scattered-light phase transition by slit edges rather than the conventional view of the transmitted-light effect by slits. The nature of the wave-particle duality of light quanta can be well understood with the help of the dynamic hypothesis of the quantized chiral photons having an intrinsic dual-energy (implicit electric field energy and hidden magnetic field energy) cyclic exchange property. With the suggested theoretical framework, the experimental diffraction pattern of the single slit is analytically determined and numerically confirmed.
\end{abstract}

PACS numbers: 42.25.-p, 42.25.Fx, 42.25.Hz

\section{INTRODUCTION}

The physical nature of light has been studied for centuries and a rich picture of the photon has been established (interference, diffraction, reflection, refraction, wavelength, frequency, speed, polarization, wave-particle duality, etc.)[1]. These findings seem to indicate that physicists have thoroughly mastered the secrets of light, unfortunately, this is not the case. From Newton and Huygens conflict about whether the light is a particle [2-5] or a wave [6] to the wave-particle duality in the quantum age [7], the debate about the nature of light has never stopped. In 1951, Einstein wrote to one of his friends: All the fifty years of conscious brooding have brought me no closer to the answer to the question, 'What are light quanta?' Of course, today every rascal thinks he knows the answer, but he is deluding himself [8].

One might have expected that with the latest developments in modern physics [9-13], the debate will eventually be resolved and a clear picture of the nature of light achieved ultimately. Over the past decades, although significant research efforts have been devoted to uncovering the features of light and photon, but the confusion status of the duality of light today is similar to or worse than that of Einstein's era. Photon, as the simplest and most elementary particle of the universe, we have to accept two contradictory pictures of wave and particle, and must use sometimes the wave picture and sometimes the particle picture, while at times we may use either. So, is the photon really as we think? As we all know, physics today is in a very embarrassing situation. On the one hand, all physical researches must rely on photons, on the other hand, we still cannot answer the question raised by Einstein. Undoubtedly, it is time for us to reconsider one fundamental question: What is the wave-particle duality of light?

In this paper, we first experimentally study the well-known single-slit diffraction and double-slit interference and obtain some very interesting results. In particular, these experiments provide strong evidence that the patterns both for single and double slits are generated by the scattered light from the edges of the slits, rather than by light transmitted through the slits as we have always considered them to be. Secondly, based on the principle of energy complementation, we propose a dualenergy (the explicit electric field energy and the hidden mag-

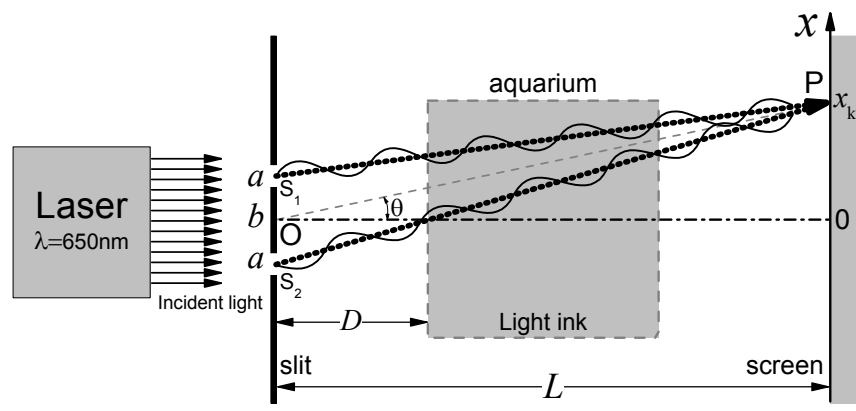

Figure 1: Schematic of improved Young's double-slit experiment. Along the light path, the aquarium can be placed anywhere behind the slit.

netic field energy) cyclic-exchange hypothesis of chiral photons with the intrinsic wave-particle duality. Under the new theoretical framework, the nature of the wave and particle property of light is unified and the formation mechanism of the single-slit diffraction and double-slit interference fringes is no longer mysterious.

\section{TWO IMPORTANT EXPERIMENTS}

Despite Young's double-slit experiment [6] (see Fig.1) has been widely regarded as providing conclusive evidence that light is a wave and was even considered by Feynman to be the heart of quantum mechanics. But it must be pointed out that what the experiment really reveals has been completely misunderstood, which may be one of the main reasons for the difficult situation of physics today. Our primary aim is to uncover the microscopic mechanism hidden in the phenomenon of interference and diffraction by designing several simple experiments. For this purpose, we have prepared a series of single and double-slit samples on 304 stainless steel plates by photochemical etching technology. 


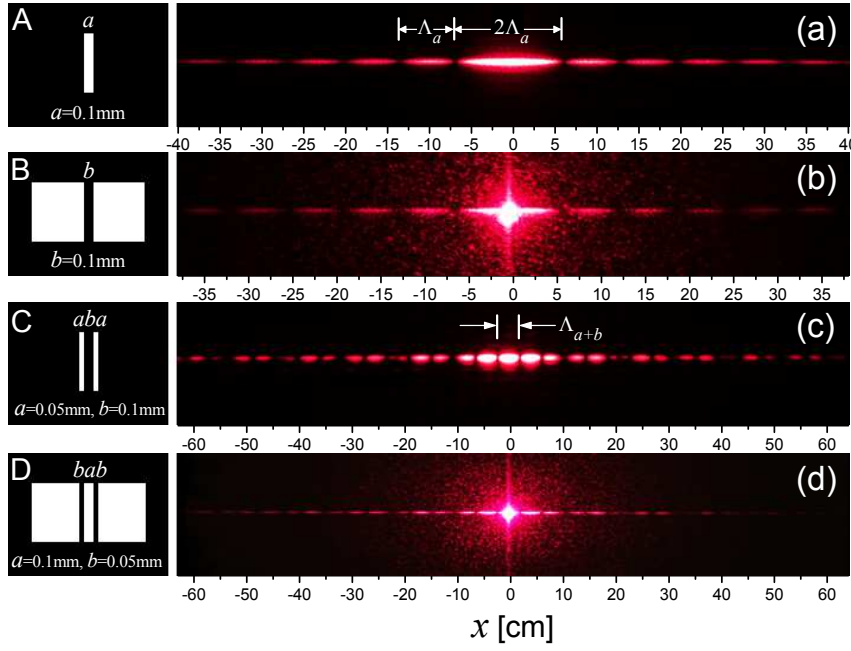

Figure 2: Comparison of diffraction and interference patterns between slits and filaments, (a) single slit, (b) single filament, (c) double slit, and (d) double filament.

\section{A. Complementary experiment}

The first experiment was carried out without placing the aquarium in the light path and the distance between the slit and the screen $L=10 \mathrm{~m}$ (see Fig. 1). Figure 2 shows the schematic of four samples (A, B, C and D) and corresponding experimental patterns (the right subgraphs), respectively. It is noteworthy that the four samples were intentionally prepared in pairs of complementary structures, which mean that if we stack samples A and B or samples C and D together, they will be completely opaque. From the right of Fig. 2, it is not difficult to find that the experimental patterns [Figs. 2(a) and 2(b), Figs. 2(c) and 2(d)] of complementary pairs are identical except for the random scattered light around the center. These experiments clearly demonstrate that the socalled interference and diffraction phenomena are actually the "edge-effect" (photon has a well-defined trajectory) instead of the conventional thinking of "slit-effect" (photon propagation without trajectory).

From Figs. 2(a) and 2(b), apart from the brightest center of width $2 \Lambda_{a}=2 L \lambda / a \approx 13.0 \mathrm{~cm}$, the stripes are changed by the period $\Lambda_{a}=L \lambda / a \approx 6.5 \mathrm{~cm}$. Why only the width of the brightest center is doubled? At the end of the Letter, we will show that this particularity is associated with the transmitted light passing directly through the slit. Furthermore, the slit-edge-scattering experimental facts suggest that the singleand double slit share the same physical mechanism. Then, the main difference between them is that the former has only two scattering edges while the latter has four, correspondingly, the pattern of the double slit has one more fringe spacing parameter $\Lambda_{a+b}=L \lambda /(a+b) \approx 4.3 \mathrm{~cm}$, as indicated in Fig. 2 (c).
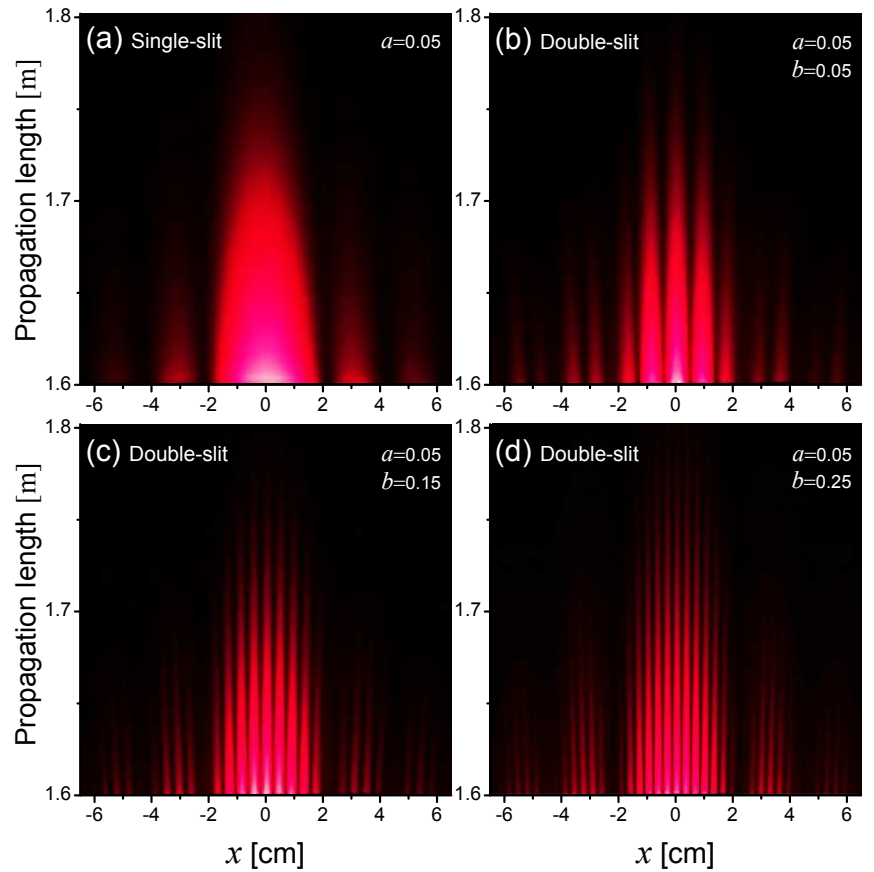

Figure 3: 3D diffraction and interference patterns of aquarium experiments, (a) single-slit of $a=0.05 \mathrm{~mm}$, (b)-(d) double-slit of $a=0.05 \mathrm{~mm}, b=0.05 \mathrm{~mm}, 0.15 \mathrm{~mm}$ and $0.25 \mathrm{~mm}$, respectively.

\section{B. 3D aquarium experiment}

To gain further physical insight into interference and diffraction phenomena, we designed the second experiment as shown in Fig. 1, where an aquarium filled with light-ink was placed at a distance of $D=1.6 \mathrm{~m}$ from the slit (Note that $D$ can be any value between 0 and $\infty$ ). Four experimental samples and corresponding slit parameters are illustrated in Fig. 3 which intuitively and vividly show the fringes and the corresponding intensity of different stripes. Incredibly, the patterns look like they were frozen in the water. According to the experimental results, we can draw the following conclusions: 1) the envelope of the double slit pattern is determined by the single slit, and the most direct effect of the double slit is the "splitting" of the single slit pattern of Fig. 3(a). From Figs. 3 (b)-(d), the splitting rules are $2(a+b) / a+1$ and $(a+b) / a$ for the central brightest stripe and the other bright stripes, respectively; 2) the red (bright) and dark stripes are 3D spatial distribution from the edges of the slits to the distant space along the direction of light propagation, instead of what we have once thought were only on the 2D screen; 3 ) both single- and double-slit experiments reveal a same collective phase transition of photons: the unstriped phase before the slits to the striped phase behind the slits.

The importance of this experiment lies in the unique way of demonstrating that the diffraction and interference fringes are $3 \mathrm{D}$ condensed state of photons extending from the edges of the slits to infinity in vacuum. So, what kind of physical mechanism can ensure that the patterns like the multiple 

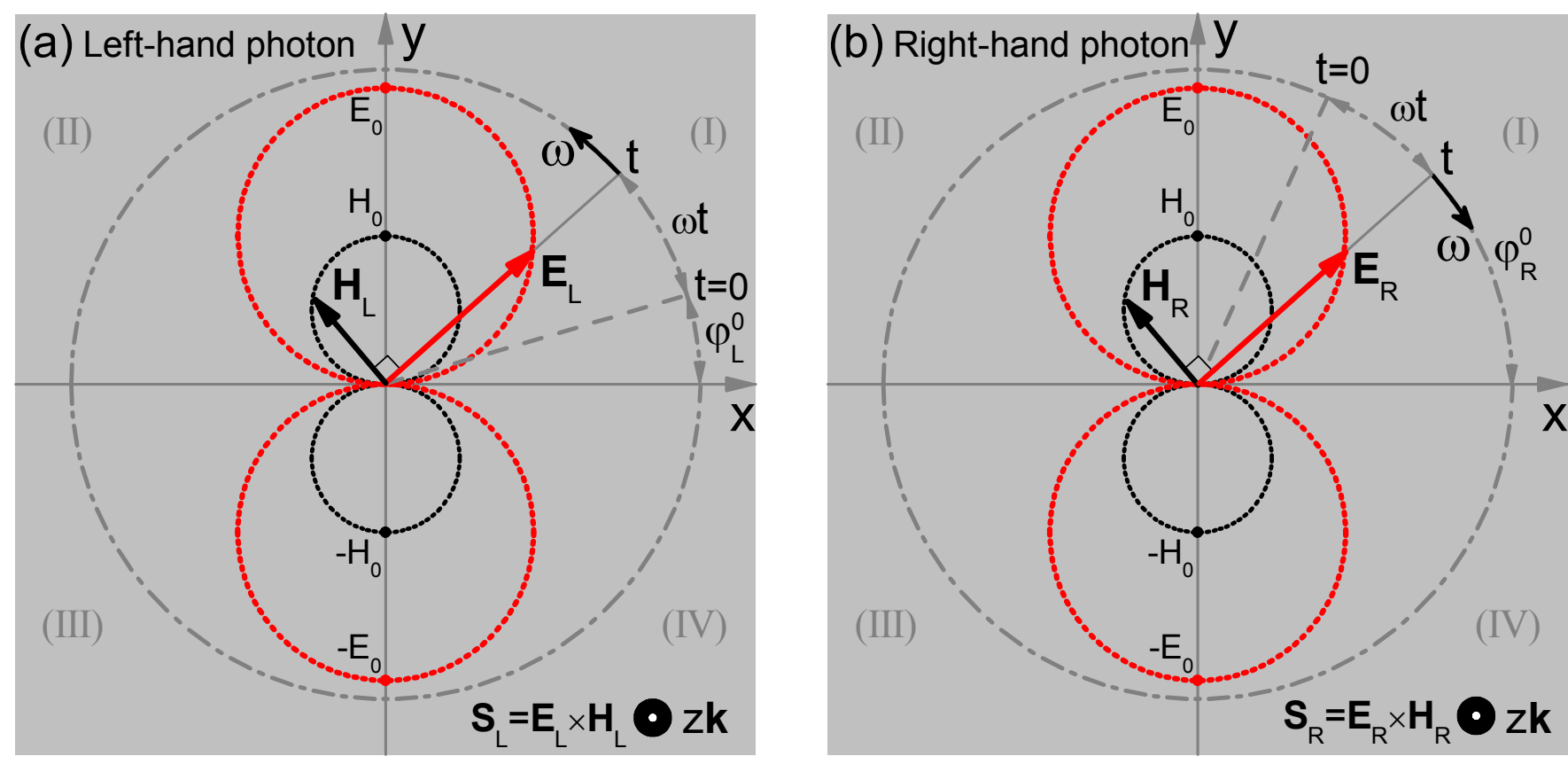

Figure 4: Schematics of chiral photons: (a) the left-handed photon rotates counterclockwise in the order of I-II-III-IV, and (b) the right-handed photon rotates clockwise in the order of I-IV-III-II. When looking at the direction of propagation of left-handed or right-handed photons, it can be found that the electric field vector $\mathbf{E}_{L}$ (or $\mathbf{E}_{R}$ ) and the magnetic field vector $\mathbf{H}_{L}\left(\right.$ or $\mathbf{H}_{R}$ ) are always perpendicular at any time $t$ and any spatial position $z$, in addition, the trajectories of the electric field vectors ( the end of red arrow) and magnetic field vectors (the end of black arrow) are two pairs of tangent circles. $\mathbf{S}_{L}$ and $\mathbf{S}_{R}$ are the Poynting vectors corresponding to the left and right-handed photons, respectively.

streams of "red photon" and "dark photon" flow in endless space and never interfere with each other?

\section{MODEL OF CHIRAL PHOTONS}

It is known that light (or photon) can be viewed as an energy particle ( $E=h \nu$, where $h$ is the Planck's constant and $\nu$ is the frequency) with zero rest mass. It can also be viewed, however, an electromagnetic wave composed of alternating electric and magnetic fields described by Maxwell's theory. We argue that these two viewpoints are incomplete because they both ignore the exact nature of the physical mechanism of how photon works internally? The propagation of wave-motion is a dynamic problem where inevitably exists an intrinsic driving force (or energy exchange mechanism) to maintain the motion. Since the wave characteristics of light have been experimentally confirmed, hence, the primary task of our research is to find out the possible hidden mechanism involves some kind of energy exchange inside the photon.

Besides. it appears as a common sense that any strict periodic wave phenomenon, such as spring oscillator, $L C$ oscillator, which are both dual-energy systems and satisfy the energy relationships: $E_{p}(t)=E_{m} \sin ^{2}\left(\omega t+\varphi_{0}\right)$ and $E_{k}(t)=$ $E_{m} \cos ^{2}\left(\omega t+\varphi_{0}\right)$, where $E_{p}(t)$ and $E_{k}(t)$ are out-phase and obey energy complementary principle: $E_{p}(t)+E_{k}(t)=$ $E_{m}=$ constant. For spring oscillator, $E_{m}=m \omega^{2} A^{2} / 2$ (where $m, \omega$ and $A$ are the mass, the frequency, and the am- plitude, respectively), while for $L C$ oscillator, $E_{m}=C U^{2} / 2$ (where $C$ is the capacitance and $U$ is the maximum voltage on the capacitance).

In the theory of electromagnetic fields, we know that the electric field $\mathbf{E}$ and the magnetic field $\mathbf{H}$ are closely related, which is stated as follows: a changing magnetic field produces an electric field and a changing electric field produces a magnetic field. Now let us assume that the proton's electric field and magnetic field energies are $E_{k}=\varepsilon_{0}|\mathbf{E}|^{2} / 2$ and $E_{p}=\mu_{0}|\mathbf{H}|^{2} / 2$, respectively, then the above statement can be represented as the energy conversion and conservation relationship: $\Delta E_{p}+\Delta E_{k}=0$ (consistent with that of oscillators). Noted that the apparent simplicity of this expression hides an important fact that $\mathbf{E}$ and $\mathbf{H}$ of photon must always keep outphase each other. However, this necessary condition is not satisfied by Maxwell's equation. From the Maxwell's equation, we have the following relationship between the electric field and magnetic field

$$
\mathbf{k} \times \mathbf{E}=\sqrt{\frac{\mu_{0}}{\varepsilon_{0}}} \mathbf{H}
$$

where $\varepsilon_{0}$ and $\mu_{0}$ are the permittivity and permeability of vacuum, respectively, $\mathbf{k}$ is the direction of the Poynting vector.

Equation (1) means that $\mathbf{E}$ and $\mathbf{H}$ are always in phase. This implies that the photon defined by Maxwell's equations does not follow the energy complementary principle. In other words, we need urgently to seek and develop new ways to study the nature of photon. As shown in Fig. 4, 
with the known scientific facts of photons (wavelength, frequency, speed, circular polarization, linear polarization, particle, wave, wave-particle duality), we propose a theoretical model of chiral photons that is more likely to help us unravel the mystery of photons. The electric field and magnetic field of the left-handed photon of Fig. 4(a) are given by

$$
\begin{aligned}
& \mathbf{E}_{L}(z, t)=E_{0}\left|\sin \left(\frac{2 \pi z}{\lambda_{0}}+\varphi_{L}^{0}\right)\right| \exp \left[i\left(\varphi_{L}^{0}+\omega t\right)\right], \\
& \mathbf{H}_{L}(z, t)=i H_{0}\left|\cos \left(\frac{2 \pi z}{\lambda_{0}}+\varphi_{L}^{0}\right)\right| \exp \left[i\left(\varphi_{L}^{0}+\omega t\right)\right]
\end{aligned}
$$

while the right-handed photon of Fig. 4(b) is defined by

$$
\begin{aligned}
& \mathbf{E}_{R}(z, t)=E_{0}\left|\sin \left(\varphi_{R}^{0}-\frac{2 \pi z}{\lambda_{0}}\right)\right| \exp \left[i\left(\varphi_{R}^{0}-\omega t\right)\right] \\
& \mathbf{H}_{R}(z, t)=i H_{0}\left|\cos \left(\varphi_{R}^{0}-\frac{2 \pi z}{\lambda_{0}}\right)\right| \exp \left[i\left(\varphi_{R}^{0}-\omega t\right)\right] .
\end{aligned}
$$

Here, $\lambda_{0}$ is the original wavelength, $\omega$ is the circular frequency, $z=c t\left(c=1 / \sqrt{\mu_{0} \varepsilon_{0}}\right.$ is the speed of light in a vacuum), and $E_{0}, H_{0}, \varphi_{L}^{0}$ and $\varphi_{R}^{0}$ are the maximum electric field intensity and magnetic field intensity, the initial phases of the left- and right-handed photons, respectively.

It must be pointed out that the proposed chiral photons are completely different from the conventional circularly polarized light. The orbit of the electric field vector of the former is a double-tangent-circle (see Fig. 4) while that of the latter is a single circle. Moreover, the double-tangent-circle indicates that the proposed chiral photons can reside in the up-state and down-state, which may be related to the spin and polarization degeneracy of photons.

\section{RELIABILITY OF HYPOTHESIS}

As a new scientific hypothesis, it must undergo a rigorous scientific evaluation to demonstrate both its reliability and its self-consistency. In the following, we will discuss from four aspects that our theory is based on a solid scientific foundation.

\section{A. Energy complementary}

The law of conservation of energy is the most important principle in the natural sciences. Needless to say, the proposed theory cannot violate the law. As seen from Eqs. (2)-(5), whether it is a left-handed photon of Eqs. (2) and (3) or a right-handed photon of Eqs. (4) and (5), it can be verified that its total energy $E_{L(R)}$ strictly follow the energy conservation relationship (or energy complementary principle):

$$
\begin{aligned}
E_{L(R)} & =\frac{\varepsilon_{0}\left|\mathbf{E}_{L(R)}(z, t)\right|^{2}}{2}+\frac{\mu_{0}\left|\mathbf{H}_{L(R)}(z, t)\right|^{2}}{2} \\
& =\frac{\varepsilon_{0} E_{0}^{2}}{2}=\frac{\mu_{0} H_{0}^{2}}{2} .
\end{aligned}
$$

\section{B. Wave equation}

Since the new hypothesis is based on the premise that it can describe the wave nature of photons, and hence its propagation must be described by the universal wave equation: $u_{t t}-c^{2} u_{z z}=0$. Also from Eqs. (2)- (5), one can find that the electric field and magnetic field of a right-handed/left-handed photon satisfy the following one-dimensional wave equations

$$
\begin{gathered}
\frac{\partial^{2} \mathbf{E}_{L(R)}(z, t)}{\partial t^{2}}-c^{2} \frac{\partial^{2} \mathbf{E}_{L(R)}(z, t)}{\partial z^{2}}=0, \\
\frac{\partial^{2} \mathbf{H}_{L(R)}(z, t)}{\partial t^{2}}-c^{2} \frac{\partial^{2} \mathbf{H}_{L(R)}(z, t)}{\partial z^{2}}=0,
\end{gathered}
$$

where $c=\omega \lambda_{0} / 2 \pi$.

\section{Linear polarization}

The linear polarization is one of the primary characteristics of light that can also be naturally derived from our hypothesis. When $\varphi_{L}^{0}=\varphi_{0}$ and $\varphi_{R}^{0}=\pi-\varphi_{0}$, a linear combination of left-handed photon of Eq. (2) and right-handed photon of Eq. (4) can be expressed by

$$
\begin{aligned}
\mathbf{E}_{L+R}(z, t) & =\mathbf{E}_{L}(z, t)+\mathbf{E}_{R}(z, t) \\
& =2 E_{0} \sin \left(\varphi_{0}+\omega t\right)\left|\sin \left(\frac{2 \pi z}{\lambda_{0}}+\varphi_{0}\right)\right| i
\end{aligned}
$$

which is a linearly polarized light along the $y$-axis direction.

\section{Wave-particle duality}

Last but the most important aspect, does our theory provide a reasonable answer to the question: what is the waveparticle duality of light? In fact, the answer is concealed in Eqs. (2)- (5). The energy complementary relationship of any chiral photon can be represented by Fig. 5. It must be pointed out that the period ( $\lambda$ in the figure) of the energy exchange is the wavelength of light measured in the experiment, which is only half of the original wavelength $\lambda_{0}$ in our theory. Moreover, it must be emphasized that usually only the electric field energy $\varepsilon_{0} E^{2}(z, t) / 2$ of the photon can induce the observable optical effects, which implies that the unused magnetic field energy $\mu_{0} H^{2}(z, t) / 2$ of the photon is a kind of hidden energy (or dark energy), and hence we can describe the single photon using only the part of the electric field energy. In this 


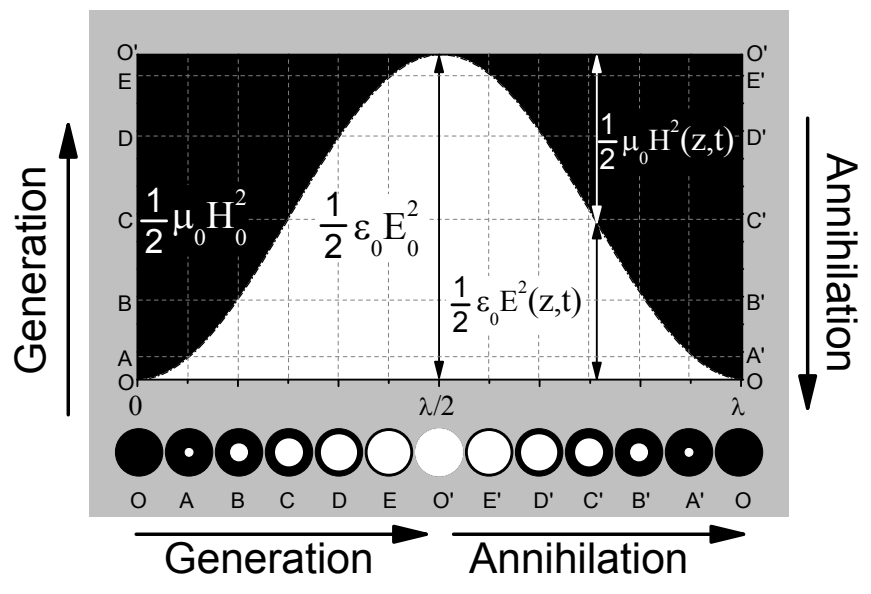

Figure 5: Graphical diagram of the electric field energy and magnetic field energy of a left-handed or right-handed photon as a function of one propagation period, where $\lambda=\lambda_{0} / 2$.

simplified picture, a single photon is an energy particle with its "size" (the electric field energy) changing periodically, as shown at the bottom of Fig. 5. In this case, we can interpret the evolution from $\mathrm{O}$ to $\mathrm{O}^{\prime}$ and from $\mathrm{O}$ ' to $\mathrm{O}$ as the generation and annihilation of a photon, respectively.

Now, what is the wave-particle duality of light? On the one hand, at any given moment the photon behaves as a particle with a definite electric field energy, on the other hand, at any time interval, the photon exhibits wave property with uncertain electric field energy, this is the physical nature of the wave-particle duality of light.

\section{APPLICATION OF NEW THEORY}

With the above theoretical and experimental researches, we now focus on the single-slit experiment that has confounded the scientific community for hundreds of years. To our knowledge, whether it is the Fresnel's half-band method of classical optics or the complicated wave function treatment of quantum mechanics [14], their researches were based on a false conjecture that the diffraction patterns are formed by transmitted light through the slit rather than the scattered light by the two edges as disclosed by our experiments of Fig.2. Furthermore, the slit-edge scattering mechanism implies that a single photon can build-up the diffraction or interference fringes.

Figure 6(a) illustrates how a single-slit (double-edges) creates the characteristic diffraction pattern on the screen. As can be seen from the figure, there are two kinds of light arriving at the screen, one is the transmitted light $I(T)$ directly passing through the slit, and the other is the scattered lights $I\left(S_{1}\right)$ and $I\left(S_{2}\right)$ from the edges $S_{1}$ and $S_{2}$ of the slit, respectively. It must be stressed again that there exists an essential difference between our theory and the traditional theories. We believe that the diffraction pattern is produced by $I\left(S_{1}\right)$ and $I\left(S_{2}\right)$ independently of $I(T)$, but most physicists insist that it is the
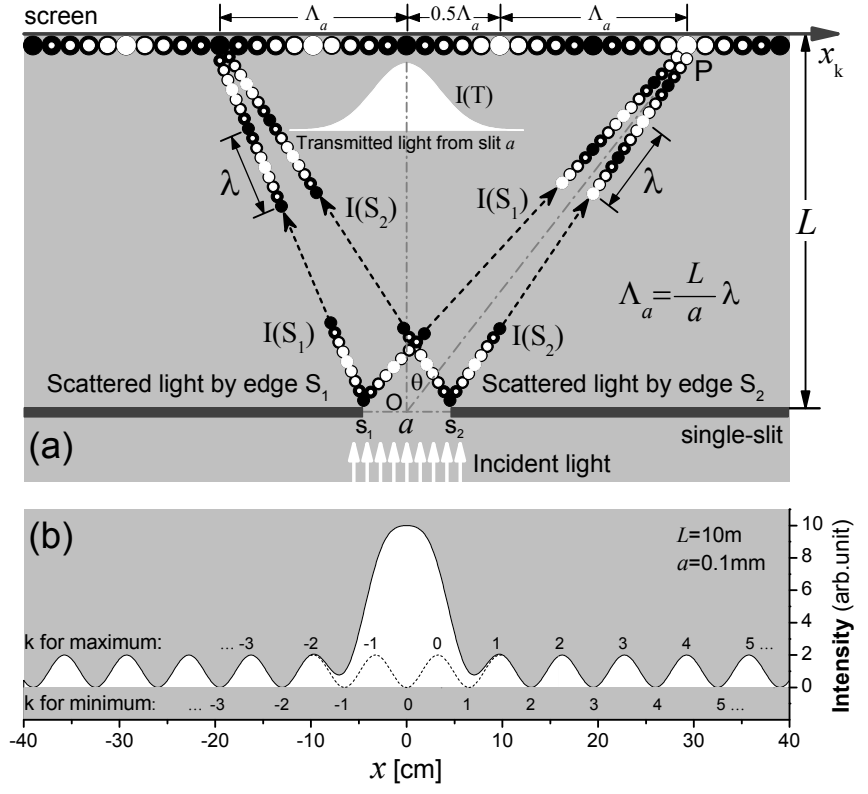

Figure 6: (a) A schematic how the bright and dark pattern built up in the single slit (double edges) experiment. (b) The numerical simulation of analytical expression (12), consistent with experimental result of Fig. 2(a).

only contribution of $I(T)$.

Without any sophisticated mathematical treatments, the present technique enables us to obtain the diffraction pattern of single-slit straightforwardly. As shown in Fig. 6(a), when the photons from edges $S_{1}$ and $S_{2}$ are both at the maximum electric field energy state, the screen at that place will appear the brightest stripe, conversely, when they are at the same maximum magnetic field energy state, the darkest stripe will appear at the corresponding position. In the approximation of $a \ll L$, the conditions for the appearance of dark and bright stripes on the screen can be respectively expressed as follows

$$
\begin{aligned}
P S_{1}-P S_{2} & \approx a \sin \theta \\
& = \begin{cases}k \lambda & k=0, \pm 1, \pm 2, \pm 3, \cdots \\
\frac{2 k+1}{2} \lambda & k=0, \pm 1, \pm 2, \pm 3, \cdots\end{cases}
\end{aligned}
$$

where $a$ is the slit width and $\theta$ is indicated in Fig. 6(a). Correspondingly, the positions of dark and bright stripes on the screen $x_{k}$ are respectively given by

$$
x_{k}=L \tan \theta= \begin{cases}k \Lambda_{a} & k=0, \pm 1, \pm 2, \pm 3, \cdots \\ \frac{2 k+1}{2} \Lambda_{a} & k=0, \pm 1, \pm 2, \pm 3, \cdots\end{cases}
$$

Eqs. (6) and (7) yield the following relationship:

$$
\frac{a \cos \theta}{L}=\frac{\lambda}{\Lambda_{a}}
$$

When the value of angle $\theta$ close to zero, thus from Eq. (8) we directly get the following familiar formula 


\section{CONCLUDING REMARKS}

$$
\Lambda_{a}=\Delta x_{k}=\left|x_{k+1}-x_{k}\right| \approx \frac{L}{a} \lambda .
$$

The total light intensity that reaches the screen consists of two parts, one is the left- and right-handed photons [Eqs. (2)(5)] scattered by the slit edges and form the periodic stripe, and the other is the direct transmitted light that does not form the stripe. By insert Eq. (9) into Eqs. (2) and (4), furthermore, if we assume that the transmitted light from the slit is a Gaussian beam, then the total light intensity on the screen can be analytically expressed as

$I=\sum_{i=1,2} I_{0}\left(S_{i}\right) \sin ^{2}\left(\pi \frac{x-x_{i}}{\Lambda_{a}}\right)+I_{0}(T) \exp \left(\frac{-2 x^{2}}{a^{2}+\Lambda_{a}^{2}}\right)$,

where $x_{i}(i=1,2)$ is the coordinate of the two edges of the slit, $I_{0}\left(S_{i}\right)$ and $I_{0}(T)$ are the maximum values of the scattered and transmitted light intensity, respectively.

Figure 6(b) shows the numerical simulation result of Eq. (10) for $I_{0}\left(S_{1}\right)=I_{0}\left(S_{2}\right)=1, I_{0}(T)=10, \lambda=650 \mathrm{~nm}$, $a=0.1 \mathrm{~mm}, x_{1}=-a / 2, x_{2}=a / 2$ and $L=10 \mathrm{~m}$, note that the parameters $a$ and $L$ are the same as those in Fig. 2(a). By comparing Fig. 2(a) with Fig. 6(b), one can see a good agreement between the experiment and the theory. In addition, the integer $k$ of Eq. (7) corresponding to the maximum and minimum of light intensity are indicated in Fig. 6(b). It should be noted that the center $x=0$ is originally the minimum becoming the maximum is caused by the superposition of the transmitted light, which in turn doubles the width of the brightest center stripe. Finally, we briefly discuss the formation of double-slit interference pattern. In our theoretical framework, the double slit (four-edges) is just a trivial extension of the single slit (two-edges). The four edges of the double slit can provide two independent interval parameters $\Lambda_{a}=L \lambda / a$ and $\Lambda_{a+b}=L \lambda /(a+b)$ with the light intensity $I_{a+b}=\sum_{i=1,4} I_{0}\left(S_{i}\right) \cos ^{2}\left[\pi\left(x-x_{i}\right) / \Lambda_{a+b}\right]$, qualitatively, the pattern is formed by merging the patterns corresponding to $\Lambda_{a}$ and $\Lambda_{a+b}$.
Based on the important experimental results of this work, we have established a new theory of the wave-particle duality of photons. Contrary to the quantum probabilistic explanation, our theory provides a unified and deterministic explanation for single- and double-slit experiments. Both theoretical and experimental results indicated that the "bright" and "dark" fringes are built-up by the electric field energy (implicit energy) and magnetic field energy (hidden energy) of the quantized chiral photons scattered by slit edges, respectively. We firmly believe that our work may shed new insights into all photon-related physical problems.

* Electronic address: xiuqing_huang@163.com

[1] M. Born and E. Wolf, Principles of Optics (Oxford: Pergamon Press, 1980), 6th ed.

[2] H. Hertz, Ann. Phys. 31, 983 (1887).

[3] A. Einstein, Ann. Phys. 17, 132 (1905).

[4] A. H. Compton, Phys. Rev. 21, 483 (1923).

[5] M. Planck, Ann. Phys. 4, 553 (1901).

[6] T. Young, Phil. Trans. R. Soc. of Lond. 94, 1 (1804).

[7] L. de Broglie, Nature 112, 540 (1923).

[8] C. Roychoudhuri, A. F. Kracklauer, and K. Creath, The Nature of Light. What is a Photon? (CRC Press, Boca Raton, FL, 2008).

[9] A. Tonomura, J. Endo, T. Matsuda and T. Kawasaki, Am. J. Phys. 57, 117 (1989).

[10] S. Dürr, T. Nonn and G. Rempe, Nature 395, 33 (1998).

[11] R. Horodecki, P. Horodecki, M. Horodecki and K. Horodecki, Rev. Mod. Phys. 81, 865 (2009).

[12] B.-G. Englert, Phys. Rev. Lett. 77, 2154 (1996).

[13] S. Kocsis, B. Braverman, S. Ravets, M. J. Stevens, R. P. Mirin, L. K. Shalm and A. M. Steinberg, Science, 332, 1170 (2011).

[14] T. V. Marcella, Eur. J. Phys. 23, 615 (2002). 